\title{
DIAGNOSTIC VALUE OF PROCALCITONIN, CRP, LEUKOCYTES AND BAL NEUTROPHILS FOR PULMONARY COMPLICATIONS IN THE IMMUNOCOMPROMISED HOST
}

\author{
Daiana Stolz, MD*, Andreas Stulz*, Beat Mueller, Prof* and Michael Tamm, Prof* \\ *Pulmonary Medicine, University Hospital Basel, Basel, Switzerland \\ \#Endocrinology, University Hospital Basel, Basel, Switzerland
}

WINNING ABSTRACT: We evaluated the diagnostic accuracy of laboratory biomarkers and BAL differential cell count for the diagnosis of bacterial infection in severe immunosuppressed patients. One-hundred and seven consecutive patients undergoing bronchoscopy for suspected pulmonary infection were included in this study. Assessment included history, clinical examination, chest image studies, CRP, procalcitonin (ProCT), leukocyte counts, and BAL results. Patients were classified as having proven, possible, and non-bacterial infection.

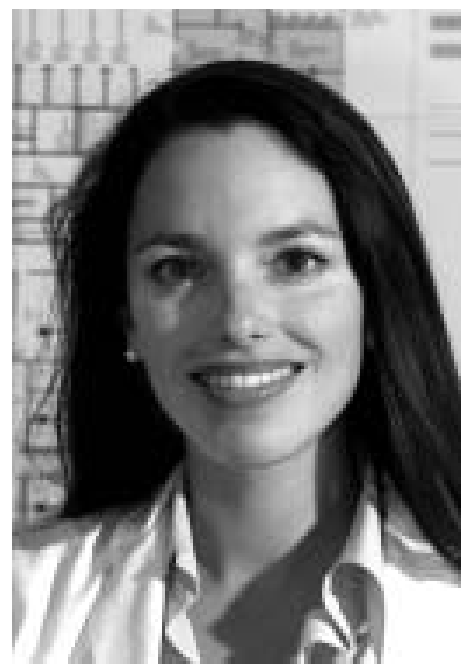

\section{Daiana Stolz}

Clinic of Pneumology, University Hospital Basel, Basel, Switzerland

\section{MY JOB AND THE UNIT IN WHICH I WORK}

This work was performed at the Clinic of Pneumology at the University Hospital Basel, Switzerland. We are a 756-bed university hospital situated close to the German and French borders. Our pneumology department is the largest in the country: we perform $>10,000$ examinations per year, including lung function tests, conventional and mobile exercise testing and sleep studies, which are offered to both ambulatory and hospitalised patients. Last year, we founded the "Lung function Academy", which offers beginner and advanced courses on lung function laboratory techniques for general practitioners and hospitals, aiming to improve the quality of lung function measurements.

We also perform several invasive procedures, including medical thoracoscopies and $>1,200$ bronchoscopies per year, including endobronchial ultrasound and interventional bronchoscopy. In order to improve the quality of care offered to patients, I have been involved in studies evaluating the preparation and execution of flexible bronchoscopy in different settings [1, 2]. Our unit also has a strong focus on basic research, investigating mechanisms of cell proliferation and differentiation in inflammatory and fibroproliferative lung diseases.

The polyvalent character of our unit enables multiple collaborative projects. A recent project examining the effect of endothelin antagonists in severe chronic obstructive pulmonary disease (COPD), for instance, involves both the Cardiology and Radiology Depts. Another example is our collaboration with the clinic of endocrinology, which allowed us to examine the usefulness of different biomarkers, particularly procalcitonin (ProCT), for the diagnosis and treatment of lower respiratory tract infection, including acute exacerbations of COPD [3]. We have also developed a long-term public health programme for smoking cessation, which has now been offered to employees of the university hospital, and Roche and Novartis, two big pharmaceutical industries based in Basel.

\section{MY WINNING ABSTRACT AS PART OF MY RESEARCH}

After finishing my training in internal and pulmonary medicine, I had the chance to attend a ward where the main focus was on the care of immunocompromised patients. I have seen a lot of pulmonary complications in haematological and post-transplant cases. Although I am in agreement regarding the indication for a diagnostic bronchoalveolar lavage before prescribing antibiotics, I was very concerned about the delay of antibiotic therapy, even for just a few hours, in this susceptible population. Therefore, I began to consider whether the biomarkers we were using to diagnose infection in immunocompetent patients could also be useful in severely immunosuppressed patients. To analyse the diagnostic value of the different clinical and laboratorial parameters to predict pulmonary bacterial infection in this setting, we decided to include all immunocompromised patients undergoing bronchoscopy with bronchoalveolar lavage (BAL) in a prospective study. This study had four aims as follows. First, we wanted to know if clinical signs help in the differential diagnosis of pulmonary complications. Secondly, we wanted to find out whether laboratory parameters, including leukocytes, Creactive protein $(\mathrm{CRP})$ and ProCT, are as useful in immunosuppressed patients as they are in the immunocompetent patient. Thirdly, we intended to examine the differential cell counts in the 


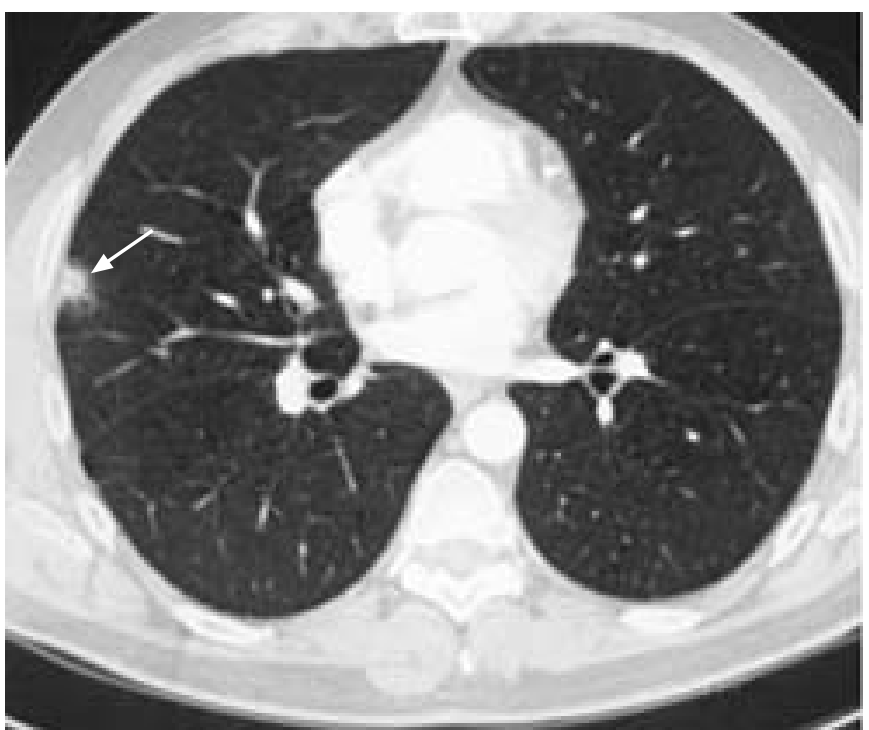

FIGURE 1. Infiltrate (indicated by the arrow) in histologically proven invasive pulmonary aspergillosis.

BAL with regard to bacterial infection. Finally, based on these analyses, we wanted to come up with a proposal to optimise the treatment algorithm in this population.

\section{MY RESEARCH AS PART OF MY WORKING GROUP/ RESEARCH TEAM}

This topic fitted well into the research area of our unit. I am part of the clinical and research team of Prof. Michael Tamm, which is currently comprised of three other attending physicians (Martin Brutsche, Werner Strobel and Jörg Leuppi), one senior molecular biologist (Michael Roth), four other part-time consultant physicians, three pulmonary fellows, three biologists, two PhD students and two research nurses. The team has been involved in many clinical and basic research projects.

The team also collaborates worldwide on projects involving biomarkers for pulmonary infection, asthma cellular mechanisms and bronchoscopic diagnostic methods. Current collaborations include work with the University of Massachusetts (Worcester, MA, USA), where I was appointed as Assistant Professor to conduct a study on ventilator-associated pneumonia, the University of Sydney (Sydney, Australia), the University of Aristotle (Thessaloniki, Greece) and the University Thoracic Clinic of Heidelberg (Heidelberg, Germany).

Data of 86 patients has been analysed so far. The mean age was 52 yrs (range 22-83 yrs); 55 were male; 32 were on high-dose chemotherapy, 18 on allogenic SZTx, 14 had had solid organ transplant, six had HIV and 16 had miscellaneous ailments. At inclusion, 65 patients (76\%) were on antibiotics; 29 (34\%) were on virostatic/fungicidal therapy. Fever was noted in 48 (56\%), cough in $51(59 \%)$, phlegm in $30(35 \%)$ and dyspnoea in 52 $(61 \%)$. In the BAL specimens, bacterial growth was documented in $23(27 \%)$ cases, viral in nine $(11 \%)$ and Pneumonocystis carinii pneumonia in three $(4 \%)$. There were localised infiltrates in 54 cases. Bacterial infection was proven in 17 patients $(20 \%)$, possible in $22(26 \%)$ and absent in $47(55 \%)$. Median ProCT values were $0.516,0.311$ and $0.085 \mathrm{ng} \cdot \mathrm{mL}^{-1}$, respectively, and ProCT, CRP and BAL neutrophils were significantly higher in the proven group as compared to the others $(p=0.001, p=0.005$ and $p<0.001$, respectively). Leukocyte counts $(p=0.293)$ were similar. The area under the curve for proven bacterial infection was 0.854 for BAL neutrophils, 0.746 for ProCT, 0.698 for CRP, 0.646 for infiltrates, and 0.601 for leukocyte counts.

In summary, BAL neutrophil counts and ProCT offer an advantage over CRP, infiltrates and leukocyte counts for diagnosing bacterial infection in immunocompromised hosts.

\section{THE IMPACT OF MY WORK ON CLINICAL OR RESEARCH PRACTICE}

This study suggests that, in immunosuppressed patients on antibiotic therapy at the time of BAL, a combination of BAL percentage neutrophils and serum ProCT may be of use to guide antibiotic therapy. This study has provided a reasonable approach to diagnosing bacterial infection in immunocompromised patients.

\section{REFERENCES}

1 Stolz D, Chhajed PN, Leuppi JD, Brutsche M, Pflimlin E, Tamm M. Cough suppression during flexible bronchoscopy using combined sedation with midazolam and hydrocodone: a randomised, double blind, placebo controlled trial. Thorax 2004; 59: 773-776.

2 Stolz D, Chhajed PN, Leuppi J, Pflimlin E, Tamm M. Nebulized lidocaine for flexible bronchoscopy: a randomized, double-blind, placebo-controlled trial. Chest 2005; 128: 1756-1760.

3 Stolz D, Christ-Crain M, Bingisser R, et al. Antibiotic treatment of exacerbations of COPD: a randomized, controlled trial comparing procalcitonin-guidance with standard therapy. Chest 2007; 131: 9-19.

4 Stolz D, Christ-Crain MM, Morgenthaler NG, et al. Copeptin, C-reactive protein and procalcitonin as prognostic biomarkers in acute exacerbations of COPD. Chest 2007; 131 1058-1067. 